\title{
Aero-engine PID parameters Optimization based on Adaptive Genetic Algorithm
}

\author{
Yinling Wang, Huacong Li \\ School of Power and Energy, Northwestern Polytechnic University, Xi'an, China
}

Keywords: ircraft engines, Genetic algorithm, Optimization.

\begin{abstract}
Study the optimization of aero-engine PID (Proportional-Integral-Derivative) controller parameters. In order to improve the tuning accuracy on aero-engine PID control parameters for the optimal solution, this paper presents a genetic algorithm-based PID parameter tuning method. Because the choice of crossover and mutation probabilities in genetic algorithm have a significant impact on the convergence speed and stability of the control system, genetic algorithm is adopted of which the crossover and mutation probability can automatically change with the fitness. Simulation results show that after variable crossover and mutation probability adaptive genetic algorithm to optimize PID control parameters, the average convergence algebra is significantly reduced and the overall control performance of the system is better.
\end{abstract}

\section{Introduction}

Aero engine work process is complex systems that big lag, nonlinear and multi-coupling throughout the flight envelope, Aero engine aerodynamic thermodynamic processes will occur a very big change, with the change of environmental conditions and flight status (airspeed, Mach number, etc.), so be quick, effective control is particularly important.

PID control is one of the earliest developed control strategy, due to its simple structure, robustness, high reliability, easy to implement and other characteristics, widely used in process control and motion control, especially in the determination system that precise mathematical model can be established. Three parameters of proportional coefficient $(K p)$, integral time $(K i)$ and derivative time ( $K d$ ) tuning are direct influenced the control effect of the PID controller, the optimizing of PID controller parameters directly affect the control effect of control system. At present, there are many ways to optimize the PID parameters, such as Ziegler-Nichols method, indirect optimization method, the gradient method, climbing method, etc. The designer of these methods, mainly through the Mathematical model of controlled object, combined with practical experience, through repeated on-site equipment debugging obtained. Because these tuning method are relatively complicated, and affected by human factors, the final parameters of being got are not usually the optimal solution. The traditional tuning method based on a specific model is not applicable, for such aero engine as complex nonlinear systems. Therefore you can take advantage of intelligent control technology for optimizing PID parameters in order to achieve optimal control.

Genetic Algorithm (GA) is an optimization method for parallel simulation of natural genetic mechanisms and biological evolution and the formation of a random search, "survival of the fittest" principle of biological evolution exist in nature, was introduced to optimize the parameters of a coded series groups.

\section{Based on GA PID parameter tuning}

Genetic algorithm is a stochastic search algorithm, which based on the principle of natural selection and genetics, is an efficient optimization method of seeking global optimal solution, not require any initialization information. Genetic algorithms aero engine PID control parameter tuning, its controller consists of two parts: one part is the traditional PID controller, as shown in Figure 1 within the dashed box. Deviation signal of systems is done calculus on proportional, integral and differential, and the weights is added processing. Another part is the GA links. Expand global 
optimization for the solution according to some technical indicators, find an optimal PID control parameters, in order to achieve the online setting.

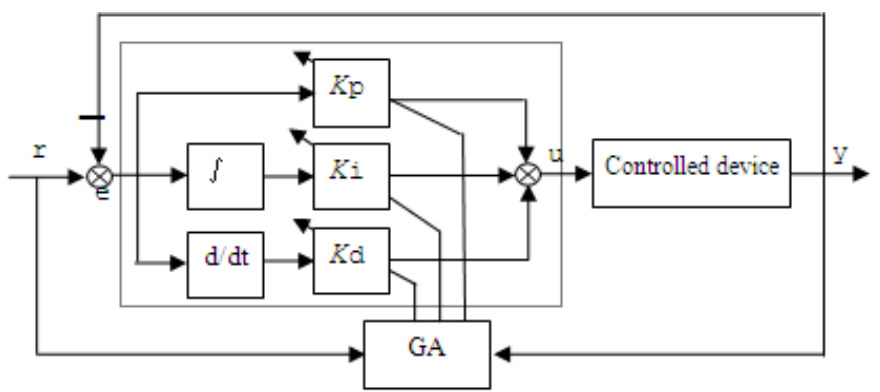

Figure1. PID control structure base on optimization

For some kind of turbofan engine, when $H=0$ and $M a=0$, establish "little deflection" dynamic model by pumping power law, the low pressure rotor speed $\left(N_{l}\right)$ for the fuel supply $\left(M_{f}\right)$ of the transfer function is that:

$$
G(s)=\frac{N_{l}(s)}{M_{f}(s)}=\frac{0.5631 s+1.434}{s^{2}+4.859 s+4.86}
$$

Input and output of the model have been normalized, the physical meaning are denoted as the percentage change in the fuel supply and the low pressure rotor speed. First give a range of the solutions of $K p, K i$ and $K d$, then the range is encoded in each solution. Range three parameters of PID controller are related to transfer function; different functions are not the same value range. Based on past experience, gives a probable of ranges of the control parameter. Where $K p$ is between 0 to 20, $K i$ is between 0 to $20, K d$ is between 0 to 1 .

The basic elements of the genetic algorithm contains on chromosome coding method, fitness function, genetic manipulation, and operating parameters. Chromosome is usually represented by a string of one-dimensional array of structures, each position corresponds to the value of each gene, and the gene is called a string consisting of a string of individual chromosomes, a certain number of individuals of the group. On the chromosome encoding method refers to the individual coding method, coding methods are divided into binary coded and real coded. Binary encoding can't reflect the structural problems, individual length great, computer memory occupied more, difficult to improve accuracy. However, real encoding is suitable for represent a wider range of values, easy to search a larger space, and deal with the constraints of more complex decision variable. In particular, can provide convenient to mix use other classical optimization methods in genetic algorithm. Therefore this paper chooses real coded.

Firstly, the genetic algorithm needs to generate the initial population. The initial population is a group of individuals randomly generated typically. Population size of genetic algorithm is not the bigger the better, because the population size is too large, directly affect the efficiency of the algorithm. The same can't be too small, because the population size is too small, may be easily lead prematurity of genetic algorithm. The number of the population is 30 in this paper.

Stability, accuracy and rapidity are the key performance indicators of measure of the controller. Optimization algorithm is looking a set of parameters to meet the conditions under the constraints. In order to obtain a satisfactory dynamic process, the time integral of absolute error performance index is used as the minimum objective function parameter selection. Squared term of the input is added to the objective function, in order to prevent excessive control. In this paper, Equation (2) expresses optimal parameters of the selected indicators.

$$
J=\int_{0}^{\infty}\left(w 1|e(t)|+w 2 u^{2}(t)\right) d t+w 3 t_{u}
$$

Where, $e(t)$ denotes the system error, $u(t)$ denotes the controller output, $t_{u}$ denotes the rise time, $w 1, w 2$ and $w 3$ denotes the weights, in order to avoid overshooting, the optimal index of system add 
on punitive function. In other words, overshoot will act as an optimal indicator when overshoot occurs. Then, optimal parameters of the selected indicators are expressed to following formula:

$$
J=\int_{0}^{\infty}\left(w 1|e(t)|+w 2 u^{2}(t)+w 4|e y(t)|\right) d t+w 3 t_{u}
$$

Where, $w 4$ denotes the weights and $w 4>>w 1$,ey $(t)$ denotes the overshooting.

Fitness function is the standard that distinguishes between good and bad groups of individuals, and is the sole basis for natural selection. The fitness function is obtained by the target function transformation. Individual fitness greater the smaller the value of the objective function, As such select the reciprocal value of the objective function as a function of individual adaptation, The formula is

$$
f=1 / J
$$

Note: To prevent $J=0$ and lead to $f=\infty, J$ should be add to a very small positive number when calculating $f$.

Genetic algorithm chooses the fittest from group of individuals by selecting the operator, high fitness individuals have a greater probability, being inherited to the next generation, and in contrast low fitness individuals have a smaller probability. Fitness proportion method is used to copy, first adaptation values are obtained through adaptation function, and then the copy probability of each string is calculated. The product of the copy probability and the each generation string is the number that the each generation string is copy in the next generation. The large copy probability lead to the large genetic probability, otherwise it will be eliminated.

Genetic algorithm can produce some new individual of unlike its parent by crossover, but for the crossover and mutation, they are searched iterative that no guidance and randomly under certain conditions the probability. The probability of crossover and mutation directly affect the convergence of the algorithm, the larger crossover probability, the faster new individuals are generated. However, the probability of crossover is too large, the possibility become greater that the genetic pattern is broken; on the contrary, the search process will slow down, so stagnant. Similarly, the probability of mutation is smaller, is difficult to generate new individuals, the algorithm will become blind aimless search, and the results will tend to be a pole, it is difficult to find suitable result. So it is very important for crossover and mutation probability of selection.

\section{Improved crossover and mutation probability algorithm}

Simple genetic algorithm (SGA) whose crossover and mutation rate are two fixed parameters, they are the known constant. Initial operation algorithms, modes focused on low fitness individuals, if a smaller crossover and mutation rate is selected, it is difficult to produce a new individual excellence of population. In algorithm running late, mode started towards the high fitness individual focus. If you continue to use the crossover rate and mutation rate of the larger, easy to destroy the good model, and cause the algorithm local convergence.

Adaptive genetic algorithm (AGA), whose main task is to design crossover and mutation probability, Let they along with the change in fitness value and evolution generations and change, In order to retain the excellent individuals of the population, to prevent the emergence of the premature phenomenon. For example, Srinvas proposed: when the fitness of individuals below the average fitness of contemporary populations, then it is poor performance of the individual, if the individual is selected in the selection mechanism, which a larger crossover and mutation rate will be used. When the fitness of individuals near maximum fitness of contemporary populations, then it is better performance of the individual, the excellent mode should be retained, even if the individual is selected in the selection mechanism, which a lower crossover and mutation rate will be used.

Figure2. The change curve of the probability of crossover and mutation was proposed by Srinvas

In the adaptive genetic algorithm that was proposed by Srinvas, The crossover and mutation probability curve is shown in Figure 2, the probability of crossover $\left(P_{c}\right)$ and the probability of mutation ( $\mathrm{Pm}$ ) are calculated according to the following formula (5) and (6) 


$$
\begin{gathered}
P C=\left\{\begin{array}{cc}
\frac{k *^{*}\left(f \max -f^{\prime}\right)}{f \max -f a v g} \quad f^{\prime} \geq \text { favg } \\
k 2 \quad f^{\prime}<\text { favg }
\end{array}\right. \\
P m=\left\{\begin{array}{cc}
\frac{k 3^{*}(f \max -f)}{f \max -f a v g} \quad f \geq \text { favg } \\
k 4 & f<\text { favg }
\end{array}\right.
\end{gathered}
$$

Where, $f$ max denotes the maximal fitness of the population, favg denotes the average fitness of the population, $f^{\prime}$ denotes the higher fitness of the two crossover individuals, $f$ denotes the fitness of mutation individual. $k 1, k 2, k 3$ and $k 4$ are constant, usually take a value between 0 to 1 .

However, the genetic algorithm is not ideal in the early evolution, because better individual in a state of almost does not change, but at this time the better individual is not necessarily the best individual global optimal solution, to increase the possibility of this easy to make the evolution toward local convergence. In evolution late, due to the initial difficult to escape from local optimal solution, eventually lead the algorithm into local convergence.

In this paper, the neuron activation function "sigmoid" is referenced. This function shows a good balance between the linear and nonlinear behavior, its image shown in Figure 3. This paper presents an improved crossover and mutation probability function, and in the early evolution, crossover and mutation probability curve slightly adjust some slow, in order to improve the close individual and. then let more excellent individuals of the population have certain and; In the evolution of late, the adaptive curve should also try to smooth some, in order to make optimum individual model has been preserved. Therefore, the probability of crossover $\left(P_{C}\right)$ and the probability of mutation $(P m)$ are calculated according to the following formula (7) and (8)
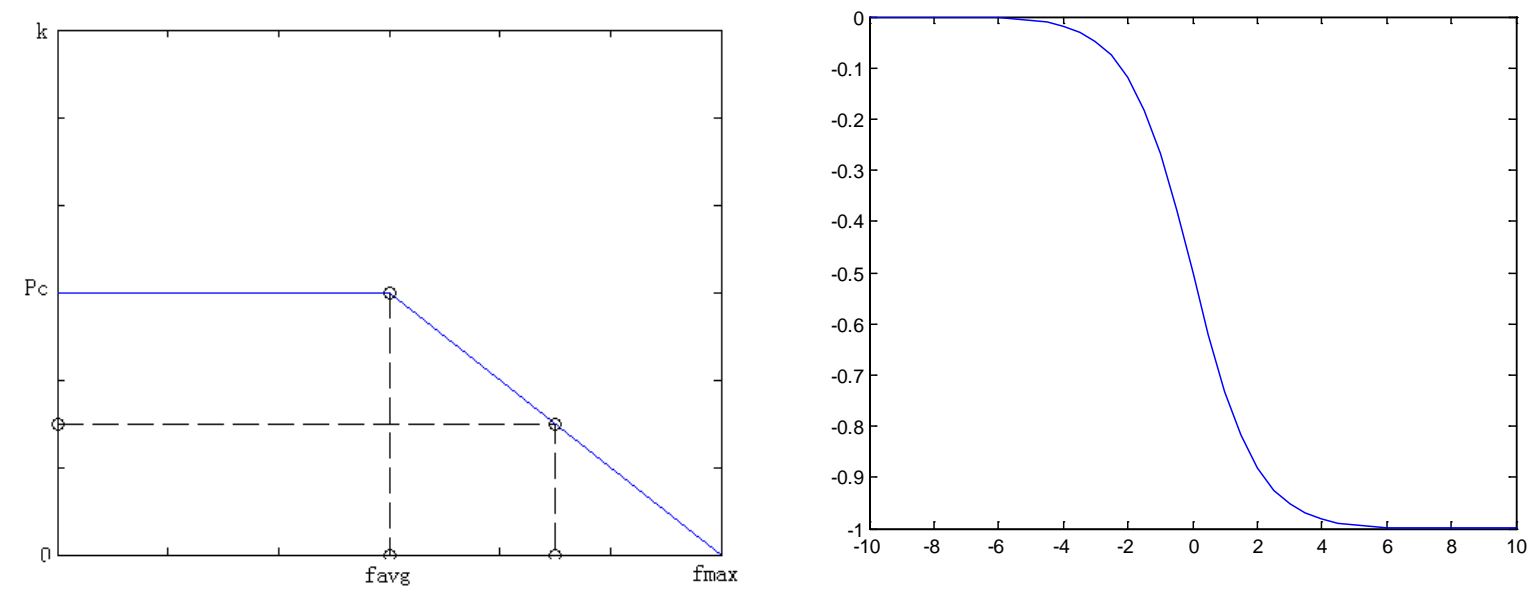

Figure3. Sigmoid function image

$$
\begin{gathered}
P C=\left\{\begin{array}{cc}
\frac{P c \max -P c \min }{1+\exp \left(L 1^{*}\left(\frac{\left(f^{\prime}-f a v g\right.}{f \max -f a v g}\right)\right)} \quad f^{\prime} \geq \text { favg } \\
P C \max \quad f^{\prime}<f a v g
\end{array}\right. \\
P m=\left\{\begin{array}{cc}
\frac{P m \max -P m \min }{1+\exp \left(L 2^{*}\left(\frac{\left(f^{\prime}-\right.\text { favg }}{f \max -f a v g}\right)\right)} & f \geq \text { favg } \\
P m \max \quad f<\text { favg }
\end{array}\right.
\end{gathered}
$$

Where, $P c \max$ and $P c$ min denotes maximum and minimum values of the probability of crossover respectively, $P m \max$ and $P m$ min denotes maximum and minimum values of the probability of mutation respectively. $L 1$ and $L 2$ are fixed constant.

Obviously, when most individuals of the population have similar fitness and average fitness, and it is close between average fitness and maximum fitness, the crossover and mutation rate of most individual is increased, and the level of increase is higher than the crossover and mutation rate of changing in accordance with the cosine. At the same time, maximum fitness nearby individuals mode 
have been retained as much as possible, bring down the crossover and mutation rate of them, Increase the probability of participate in a cross pattern pairing for the individual, strive to make the algorithm to escape from local convergence.

\section{Simulation experiments}

The algorithm was verified by some kind of turbofan engine, in order to verify the effectiveness of the algorithm.

On the one hand, the crossover probability and mutation probability calculation functions used in this paper, the PID parameters of the adaptive genetic algorithm engine tuning, to verify the validity of the method. Where $L 1=19.786, L 2=21.352$, Crossover probability $P c \min =0.5, P c \max =0.9$, Mutation probability Pm min $=0.02$, Pm max $=0.05$, Population size of 30, inherited 100 generations. Optimal index curve of weighting algorithm is illustrated in Figure 4, get a group of PID parameters. Where, the proportionality coefficient $K p=12.4494$, Integration time $K i=13.2388$ and Differential time $K d=0.7075$, closed-loop system unit step response is illustrated in Figure 5.

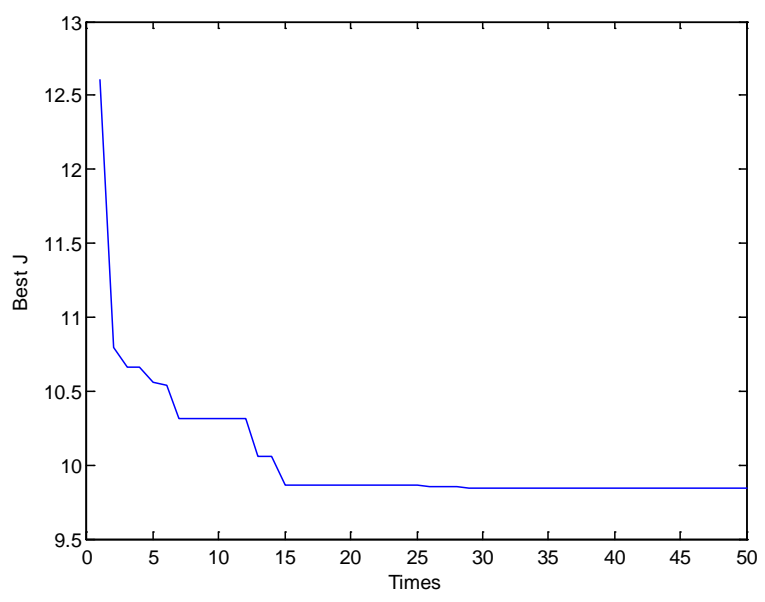

Figure4. Optimal index curve of weighting algorithm

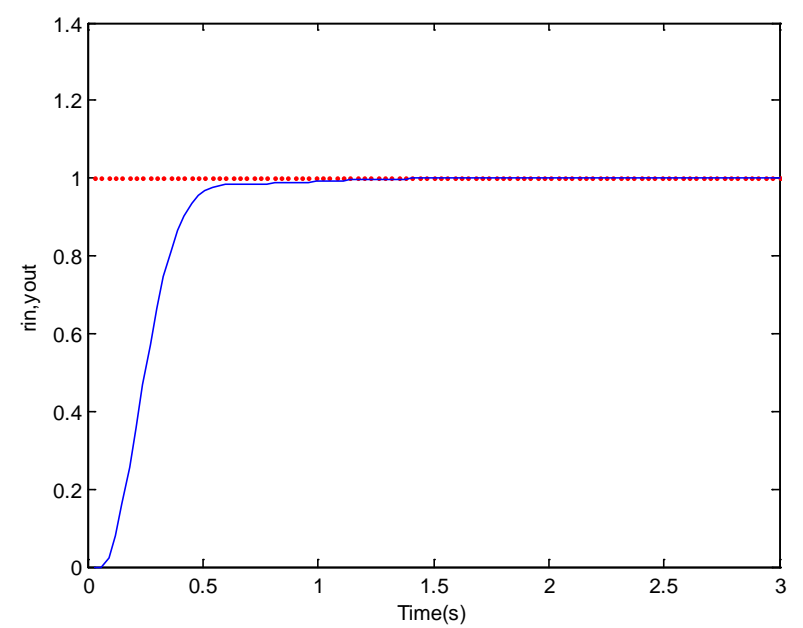

Figure5. Closed-loop system unit step response

On the other hand, first, the use of standard genetic algorithm SGA.

Where Crossover probability $P c=0.9$, Mutation probability $P m=0.05$; Secondly, the use of adaptive genetic algorithm AGA which Srinvas proposed, where $k 1=0.5, k 2=0.9$, $k 3=0.02, k 4=0.05$; Finally, the use of IAGA adaptive genetic algorithm which this paper proposed. Using Equation (1) of the test model, running operation was repeated 1000 times. Add up 
the convergence algebra which every optimal performance index and no more than $0.1 \%$ error of first arrived. The average convergence algebra of SGA, AGA and IAGA are show in Table 1.

Table1. The average convergence algebra of different approaches compare

\begin{tabular}{cccc}
\hline No. & Type & Population size of Solutions & Average convergence algebra \\
\hline 1 & SGA & 30 & 25.563 \\
2 & AGA & 30 & 20.432 \\
3 & IAGA & 30 & 16.455 \\
\hline
\end{tabular}

As can be seen from Table 1, the average convergence algebra is significantly reducing, when aero engine PID control parameters are tuned by the adaptive genetic algorithm, which the crossover and mutation probability are variable.

\section{Conclusions}

This paper analyzes the problem of aero engine PID parameters tuning, and proposes a genetic algorithm optimization PID parameter tuning method. Analyzed of the shortcomings of current traditional genetic algorithm, presented a genetic algorithm which the crossover and mutation probability are variable. Through the use of different algorithms for PID tuning parameters and simulation, the results show that using the proposed algorithm for PID tuning parameters of aircraft engines has the following advantages: the setting time is short, the population average convergence algebra change small, adjust the effect is better, etc. The algorithm is particularly suitable for this complex system of aero engine, and can maximize the potential performance of aircraft engines.

\section{References}

[1] Chang, Di. \& Li, Hua-Cong. 2009. Application of fuzzy neural network controller based on GA in aero-engine control. Computer Simulation, 26(10):65-68.

[2] Cong, S. \& Lang, Y. 2009. PID-like neutral network nonlinear adaptive control for uncertain multivariable motion control systems, IEEE Transaction on industrial Electronics, 10(56):3872-3879.

[3] Fan, Si-Qi. 2008. Aircraft engine control, Xi'an: Northwestern University Press.

[4] Gong, Chun. \& Wang, Zheng-Lin. 2009. Proficient matlab optimization calculation, Beijing: Electronic Industry Press.

[5] Jin, Jing. \& Su, Yong. 2005. An improved adaptive genetic algorithm. Computer Engineering and Applications, 18:64-69.

[6] Li ,Yan-Mei. 2012. An improved genetic algorithm and its application. Guangzhou: South China University of Technology.

[7] Liu, Jin-Kun. 2007. Advanced PID control the matlab simulation, Beijing: Electronic Industry Press. 